\title{
Fermi surface and electronic structure of $\mathrm{Pb} / \mathrm{Ge}(111)$
}

\author{
A. Mascaraque \\ Departamento de Física de la Materia Condensada and Instituto Universitario de Ciencia de Materiales "Nicolás Cabrera," \\ Universidad Autónoma de Madrid, 28049 Madrid, Spain \\ J. Avila \\ Instituto de Ciencia de Materiales, CSIC, 28049 Madrid, Spain \\ and LURE, Bâtiment 209D, Université Paris-Sud, F-91405 Orsay, France \\ E. G. Michel \\ Departamento de Física de la Materia Condensada and Instituto Universitario de Ciencia de Materiales "Nicolás Cabrera," \\ Universidad Autónoma de Madrid, 28049 Madrid, Spain \\ M. C. Asensio \\ Instituto de Ciencia de Materiales, CSIC, 28049 Madrid, Spain \\ and LURE, Bâtiment 209D, Université Paris-Sud, F-91405 Orsay, France \\ (Received 7 August 1997; revised manuscript received 27 January 1998)
}

\begin{abstract}
The electronic structure of $\mathrm{Pb} / \mathrm{Ge}(111)$ has been probed along the temperature-induced phase transition $\alpha$ $-\sqrt{3} \times \sqrt{3} R 30^{\circ} \Rightarrow 3 \times 3$ using angle-resolved photoemission. The $\alpha-\sqrt{3} \times \sqrt{3} R 30^{\circ}$ phase is metallic due to the existence of a half-filled, dispersing surface band. The $3 \times 3$ phase is characterized by the appearance of an additional surface band with $3 \times 3$ periodicity, whose role in the phase transition is discussed. The Fermisurface topology of the $\alpha-\sqrt{3} \times \sqrt{3} R 30^{\circ}$ phase has been probed using angle-resolved photoemission. Its shape is undulated, and it resembles strongly the theoretical prediction, with a Fermi momentum of $0.31 \AA^{-1}$ along $\overline{\Gamma K}$ directions and $0.40 \AA^{-1}$ along $\overline{\Gamma M}$ directions. These values were determined from different experimental methods, and agree with the values needed for a perfect $3 \times 3$ nesting. However, the Fermi surface exhibits no large flat areas suitable for electronic nesting.

[S0163-1829(98)03520-6]
\end{abstract}

\section{INTRODUCTION}

In a wide range of low-dimensional systems a chargedensity wave (CDW) is stabilized at low temperatures. ${ }^{1,2}$ The origin of the CDW phase transition in a metal lies in an instability of the Fermi surface (FS), which involves a strong electron-phonon interaction. ${ }^{1,3}$ A CDW is stabilized whenever sizable nesting between flat areas of the FS is possible. ${ }^{3}$ In this case, the symmetry of the new unit cell is determined by the nesting vector, which is equal to twice the Fermi momentum $\left(k_{F}\right)$. In fact, most of the peculiar properties of CDW materials are related to the topology of their FS's. In addition to bulk CDW materials, the formation of a surface CDW has been proposed in a variety of surface systems, ${ }^{4,5}$ including $\mathrm{W},{ }^{6-10}$ and alkali metals on $\mathrm{Cu}(111) .{ }^{11}$ Recently, Carpinelli et al. ${ }^{12}$ have reported conclusive scanning tunneling microscopy (STM) and low-energy electron diffraction (LEED) results, which show a surface phase transition from the room-temperature $(300 \mathrm{~K}, \mathrm{RT}) \alpha-\sqrt{3} \times \sqrt{3} R 30^{\circ} \mathrm{-Pb} /$ $\mathrm{Ge}(111)$ phase $^{13-17}$ (in the following $\alpha-\sqrt{3}$ ) to a $3 \times 3$ structure at low temperature $(100 \mathrm{~K}, \mathrm{LT})$. They proposed that the structural phase transition is due to the stabilization of a surface CDW. The origin of the CDW would lie in the existence of suitable $3 \times 3$ nesting vectors in the $\alpha-\sqrt{3}$ Fermi surface (see below and Ref. 12).

We set out in this work to investigate the FS topology of the $\alpha-\sqrt{3}-\mathrm{Pb} / \mathrm{Ge}(111)$ phase using angle-resolved photoemission. In the case of bulk materials, FS topology can be studied using different techniques (e.g., de Haas-van Alphen effect). In contrast, the investigation of the FS of systems with reduced dimensionality is easily performed using angleresolved ultraviolet photoemission (ARUPS). ${ }^{5,18-20}$ This technique directly probes the electronic states at the Fermi energy $\left(E_{F}\right)$ with momentum resolution, and thus it allows one to obtain a full plot of the FS and the value of $k_{F}$. Our results show that at RT, the FS of the metallic $\alpha-\sqrt{3}$ structure presents an undulated shape that agrees well with theoretical predictions. ${ }^{12}$ The value of $k_{F}$ along $\overline{\Gamma K}$ and $\overline{\Gamma M}$ directions was precisely determined using three different methods. The values found are compatible with a $3 \times 3$ nesting along $\overline{\Gamma K}$ directions. However, the FS does not present a large nesting along these directions, since there are no flat areas connected by $2 k_{F}$ vectors.

On the other hand, Carpinelli et al. ${ }^{12}$ found that the 3 $\times 3$ phase was semiconducting, a result that could not be explained by theoretical calculations in the local-density approximation (LDA), and was attributed to electron correlation effects in the low-temperature (LT) phase. Goldoni, Cepek, and Modesti ${ }^{21}$ have recently detected the band gap opening at low temperature using angle-resolved photoemission, but they could not find any dispersing surface-state band crossing $E_{F}$. This is an important requirement in the 
model exposed above for the transition, and is also expected from theoretical calculations. ${ }^{12,22}$ In fact, the metallic character of the $\alpha-\sqrt{3}$ phase is due to the existence of a halffilled surface-state band ${ }^{12}$ (see also below). In an effort to understand the nature of both RT and LT phases, we have analyzed the electronic band structure of both phases. We show that the $\alpha-\sqrt{3}$ phase presents a dispersing surface band that crosses $E_{F}$, and explains the metallic character of this phase. In a simple band picture, the new $3 \times 3$ periodicity formed at LT should produce band folding. These kinds of effects are indeed observed, but in addition to them several other striking changes take place in the vicinity of $E_{F}$. First, a new surface band with $3 \times 3$ periodicity is observed at LT close to $E_{F}$. Concomitantly with the phase transition and the opening of a pseudogap, ${ }^{12,21,23}$ a narrow peak close to $E_{F}$ appears. We interpret this peak as a result of partial electron localization. . All these features of the $3 \times 3$ reconstruction are reversible with temperature, and evidence strong modifications in the two-dimensional electronic structure of $\mathrm{Pb} / \mathrm{Ge}(111)$ along the phase transition.

\section{EXPERIMENT}

The experiments were performed in an ultrahigh vacuum (UHV) chamber (base pressure: $5 \times 10^{-11} \mathrm{mbar}$ ) equipped with an angle-resolving hemispherical analyzer mounted in a UHV goniometer and a high-precision manipulator that permits rotation in the full $360^{\circ}$ azimuthal emission angle $(\Phi)$ and $90^{\circ}$ polar emission angle, relative to the surface normal $(\theta)$. We measured conventional angle-resolved energydistribution curves, but photoelectron intensities were also monitored in a full $2 \pi$ sr solid angle by fixing a kinetic energy for detection in the electron analyzer, defined as absolute counts above zero level. The sample was then rotated around its normal in the azimuthal direction and the photoelectron intensity was recorded every $1^{\circ}$ at fixed $\theta$, with an absolute angular precision of $0.5^{\circ}$ for both $\theta$ and $\Phi$. The relative values of $\theta$ were monitored from the reading of the analyzer goniometer, since the surface normal direction was kept constant during the experiment (constant polarization vector, angle of incidence of light: $45^{\circ}$ ). The absolute values of both angles were calibrated using the dispersion of both bulk $\mathrm{Ge}(111)$ and surface $\alpha-\sqrt{3}$ peaks. In this way we map a constant energy contour that coincides with the FS if the right kinetic energy is selected (see also below). The overall energetic resolution was $100 \mathrm{meV}$ at $h \nu=32 \mathrm{eV} . E_{F}$ was measured from a Ta foil in electrical contact with the sample and close to it. The sample work function was measured from the low-energy cutoff in the photoemission spectra. The experiments were performed using synchrotron light from the SU6 beamline of the Super-Aco storage ring in Orsay at the Spanish-French station. In all experiments, a Ge(111) sample was sputtered with $\mathrm{Ar}$ and heated up to $650^{\circ} \mathrm{C}$ for several minutes by resistive heating, and then cooled down to RT slowly in order to get a clean $\mathrm{Ge}(111) c(2 \times 8)$ surface. $\mathrm{Pb}$ coverage $\left(\Theta_{\mathrm{Pb}}\right.$, accuracy $\left.\pm 10 \%\right)$ was measured with a quartz microbalance and cross checked by a quantitative comparison of $\mathrm{Pb} 5 d$ and $\mathrm{Ge} 3 d$ core levels. ${ }^{24}$ We define
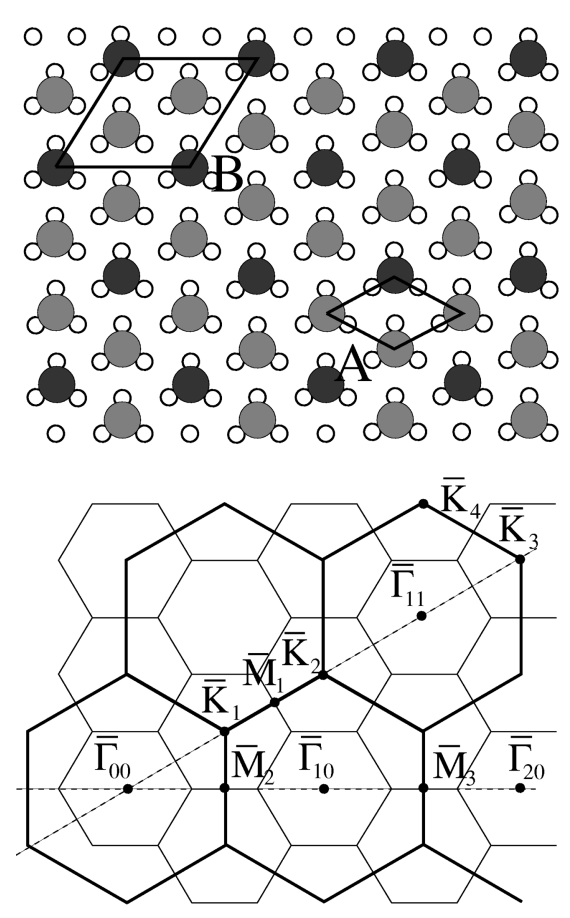

FIG. 1. Top: schematic representation of the $\alpha-\sqrt{3}$ (A) and 3 $\times 3$ (B) unit cells. Open circles, Ge atoms. All dark circles correspond to equivalent $\mathrm{Pb}$ atoms in the $\alpha-\sqrt{3}$ reconstruction. The different shading patterns denote a possible inequivalence to produce a LT $3 \times 3$ phase. Bottom: reciprocal space of both structures ( $\alpha-\sqrt{3}$, bold lines; $3 \times 3$, thin lines).

$\Theta_{\mathrm{Pb}}=1 \mathrm{ML}$ as an amount of atoms equal to the $\mathrm{Ge}(111)$ surface density $\left(7.21 \times 10^{14}\right.$ atoms $\left./ \mathrm{cm}^{2}\right)$.

\section{THE $\alpha-\sqrt{3}$ PHASE: RESULTS AND DISCUSSION}

The $\alpha-\sqrt{3}$ phase of $\mathrm{Pb} / \mathrm{Ge}(111)$ corresponds to occupation of $T_{4}$ sites of the $\mathrm{Ge}(111)$ surface by $\frac{1}{3} \mathrm{ML} \mathrm{Pb}$ atoms ${ }^{13-17,25-27}$ (see Fig. 1). This phase reverts to $3 \times 3$ at LT. ${ }^{12}$ A $3 \times 3$ superstructure could be achieved after a small collective distortion of $\mathrm{Pb}$ atoms, as illustrated in Fig. 1 (see also Ref. 12). For coverages below $\frac{1}{3}$ ML, the existence of a mosaic phase has been reported. ${ }^{28,29}$ In this phase the missing $\mathrm{Pb}$ atoms to complete an $\alpha-\sqrt{3}$ reconstruction are replaced by intermixed Ge atoms. ${ }^{29}$ The formation of the mosaic phase gives rise to distinct features in the valence band. ${ }^{28,30}$ Intermixed Ge adatoms have been observed by STM in a rather wide range of coverage. ${ }^{16,29} \mathrm{We}$ have found that the process of intermixing between $\mathrm{Ge}$ and $\mathrm{Pb}$ is facilitated when $\mathrm{Pb}$ is evaporated at RT or above. In this work, $\mathrm{Pb}$ was evaporated at LT, and later on heated up to RT. In these conditions the characteristic features of the mosaic phase were not observed in photoemission, and we believe that larger-sized $\alpha-\sqrt{3}$ domains of higher perfection are grown by this method, probably because $\mathrm{Ge}$ intermixing is hindered. ${ }^{31}$

The $\alpha-\sqrt{3}$ phase presents two sets of surface bands. ${ }^{28,32}$ The lower surface band $S_{1}$ at $\sim 1 \mathrm{eV}$ binding energy (BE) [see Fig. 2 (left)] comes from $\mathrm{Pb}$ backbonding (mainly through $p_{x}, p_{y}$ orbitals) with substrate dangling bonds. The upper surface band $S_{2}$ has a stronger $\mathrm{Pb} p_{z}$ character. The spectra shown in Fig. 2 demonstrate that both surface bands 


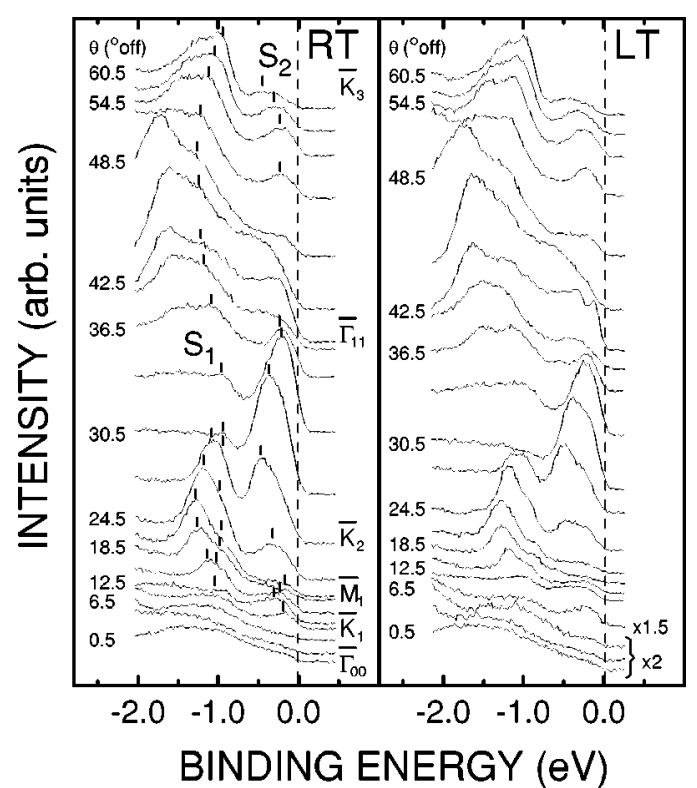

FIG. 2. Selected photoemission spectra as a function of emission angle along the $\overline{\Gamma K}$ direction $(h \nu=32 \mathrm{eV})$ at RT (left) and LT (right). The angular range corresponds to the area between $\bar{\Gamma}_{00}$ and $\bar{K}_{3}$ in Fig. 1 .

are dispersing, indicating long-range order in the surface, and a significant degree of delocalization in the valence electrons. ${ }^{12,22,32,33}$ A simple electron counting shows that the number of valence electrons in the $\alpha-\sqrt{3}$ unit cell (7) is odd. Thus, $S_{2}$ is half-filled and the surface should be metallic. Figure 3 (top) shows the dependence of BE versus parallel momentum along the $\overline{\Gamma K}$ direction of the surface Brillouin zone at RT. $S_{2}$ has a minimum BE at the $\bar{K}$ points and disperses towards $E_{F}$ when going away from the $\bar{K}$ points towards the $\bar{\Gamma}$ points. The peak is observed only in part of the Brillouin zone, suggesting that it crosses $E_{F}$ between the $\bar{\Gamma}$ and $\bar{K}$ points, and also between the $\bar{K}$ and $\bar{M}$ points (see also below, and Ref. 12). Figure 4 (top panels) shows in more detail the dispersion of $S_{2}$ near $E_{F} \cdot{ }^{34}$ Note that as the surface band disperses through $E_{F}$, the intensity detected at $E_{F}$ diminishes accordingly. This photoelectron intensity can thus be used to monitor the crossing process. ${ }^{5,18,19,35}$ Figure 4 (bottom panels) shows the intensity integrated in the window between $E_{F}-0.05 \mathrm{eV}$ and $E_{F}+0.05 \mathrm{eV}$ as a function of parallel momentum $\left(k_{\|}\right){ }^{36}$ The data points correspond to the spectra shown above. The symmetry points of the $\alpha-\sqrt{3}$ surface Brillouin zone have been marked. The larger maxima of the intensity are usually attributed to band crossing, ${ }^{5}$ and thus they determine the value of $k_{F}$. From this plot we obtain values for the crossing point of $1.475 \pm 0.03 \AA^{-1}$ and $2.15 \pm 0.03 \AA^{-1}$ along $\overline{\Gamma K}$. From these values a mean $k_{F}$ (reduced to the first Brillouin zone) of $0.34 \pm 0.03 \AA^{-1}$ is obtained. A similar analysis along the $\overline{\Gamma M}$ direction shows that the distance between symmetric maxima is $0.25 \AA^{-1}$ (Fig. 4). $k_{F}$ (reduced to the first Brillouin zone) is 0.40 $\pm 0.03 \AA^{-1}$. The error bars were estimated considering both the goniometer accuracy and the error in determining the crossing point from Fig. 4. The different values along the

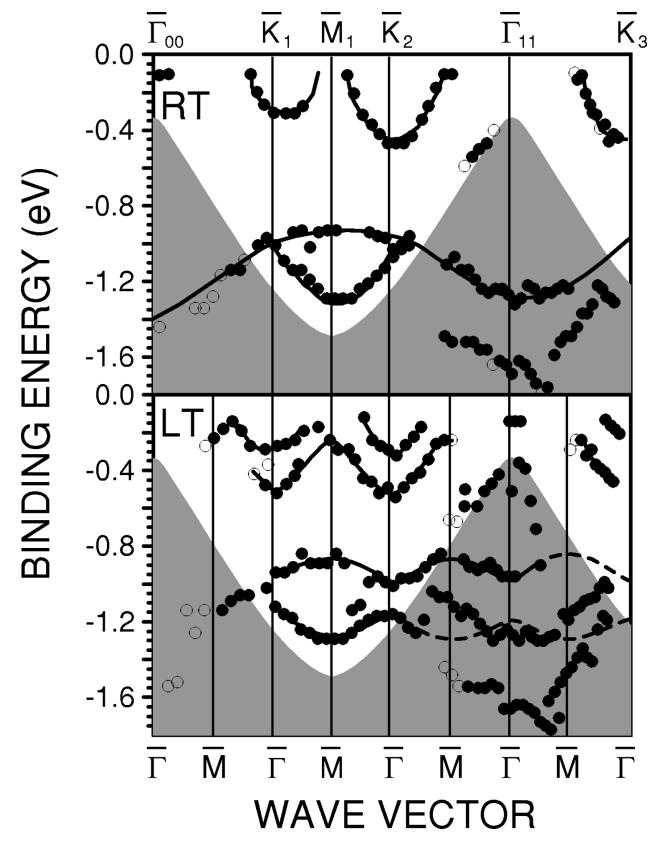

FIG. 3. Top: Binding energy from $E_{F}$ vs parallel momentum for the surface bands closer to the Fermi energy in the $\alpha-\sqrt{3}$ phase. The continuous line is a guide to the view. The unshaded area corresponds to the gap projected by bulk Ge bands. Bottom: as panel (a) for the $3 \times 3$ phase. Dashed lines indicate areas where the expected $3 \times 3$ folding is not observed. Symmetry points of the $\alpha$ $-\sqrt{3}(3 \times 3)$ phase are shown in the upper (lower) axis. Note the band splitting around the $\bar{K}$ points at LT.

$\overline{\Gamma M}$ and $\overline{\Gamma K}$ directions demonstrate that the FS is undulated, with a larger $k_{F}$ along the $\overline{\Gamma M}$ directions. A secondary maximum is found along the $\overline{\Gamma K}$ directions at $\sim 1.8 \AA^{-1}$ (which coincides with $\left.\bar{\Gamma}_{11}\right) \cdot 37$

Figure 5 displays the dispersion of the surface band versus parallel momentum along the $\overline{\Gamma K}$ direction. The data points have been fitted using a parabola, which reproduces quite well the shape of the dispersion curve, including the minimum of the BE around $\bar{K}$ points. From this fit we determine a mean value for $k_{F}$ of $1.51 \pm 0.02 \AA^{-1}$, which gives $0.30 \pm 0.02 \AA^{-1}$ reduced to the first Brillouin zone. In a previous publication, Carpinelli et al. ${ }^{12}$ have shown firstprinciples density-functional calculations within the localdensity approximation for the dispersion of $S_{2}$ in the $\alpha$ $-\sqrt{3}$ phase. ${ }^{12}$ Our experimental data at RT are in good agreement with their theoretical calculations. The dispersion of $S_{2}$ agrees also well with other density-functional theory calculations for this surface. ${ }^{22} \mathrm{We}$ conclude that the electronic band structure of the $\alpha-\sqrt{3}$ phase is well explained within one-electron theories. These results agree well with previous experimental evidence, ${ }^{28}$ but disagree with more recent results, ${ }^{21}$ where no dispersing surface bands were found. The lack of dispersion was attributed to inadequacies of the LDA approximation to describe the $\alpha-\sqrt{3}$ phase. We speculate that the different surface preparation method employed in Ref. 21 ( $\mathrm{Pb}$ deposition at elevated temperature) might give rise to smaller $\sqrt{3}$ domains due to Ge intermix- 


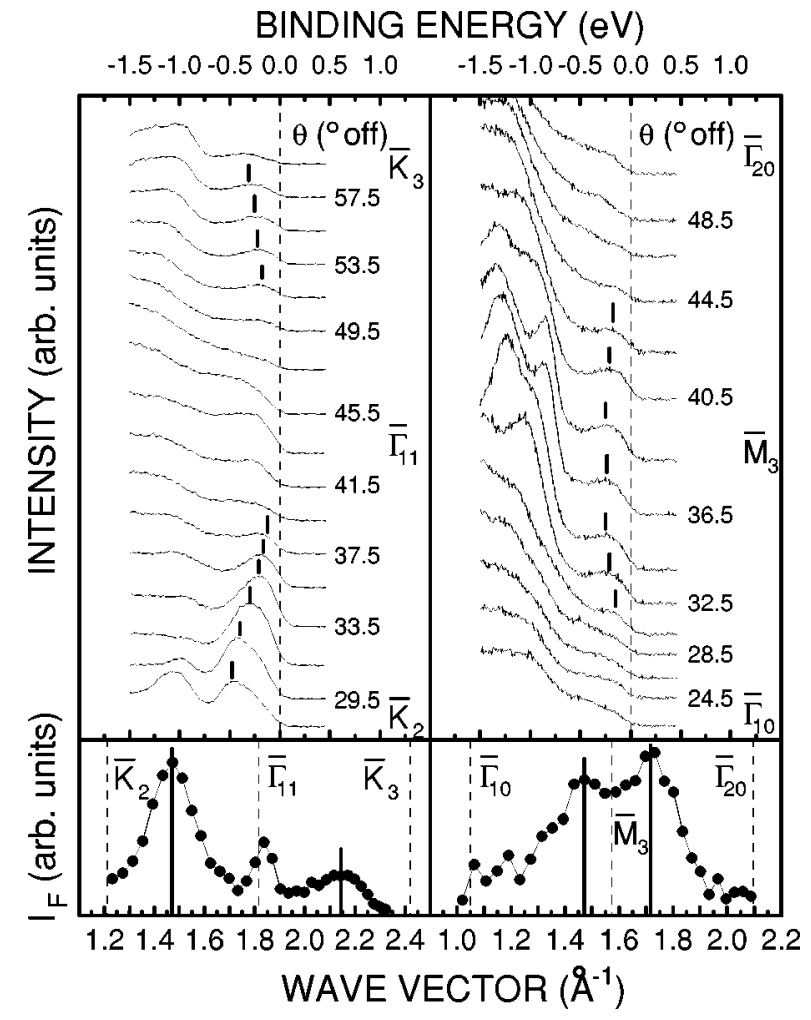

FIG. 4. Top panels: photoemission spectra taken with $h \nu$ $=32 \mathrm{eV}$ (incidence angle of light $45^{\circ}$ ) vs emission angle $\theta$ along the $\overline{\Gamma K}$ direction (left) and the $\overline{\Gamma M}$ direction (right). Note in both cases the dispersion of the surface band marked with ticks. Bottom: intensity in a window of $0.1 \mathrm{eV}$ around $E_{F}$ measured from the spectra shown in the upper panel ( $\overline{\Gamma K}$ and $\overline{\Gamma M}$ directions). The main maxima (bold lines) correspond to crossing of $E_{F}$, and are used to determine the value of $k_{F}$.

ing, and thus to a decrease of the long-range order of the $\alpha$ $-\sqrt{3}$ phase.

Figure 6 (top) shows the distribution in reciprocal space of the photoelectron intensity at $E_{F}$, and is a graphical twodimensional image of the FS. ${ }^{18,38}$ This view is specially simple in a two-dimensional system as $\mathrm{Pb} / \mathrm{Ge}(111)$, since the FS is in fact a line. ${ }^{5,12,18}$ Figure 6 (bottom) is a schematic representation of the theoretical FS topology from the calculation by Carpinelli et al. ${ }^{12}$ For an easier comparison with the experiment, the unoccupied side of the FS has been shaded. The experimental FS presents an undulated shape, with a $k_{F}$ larger along the $\overline{\Gamma M}$ directions than along $\overline{\Gamma K}$, in agreement with theoretical predictions, ${ }^{12}$ and the results exposed above. This is evidenced by the wavy aspect of the inner and outer parts of the intensity around the first $\alpha$ $-\sqrt{3}$ Brillouin zone in Fig. 6 (top).

The analysis of FS images as obtained by ARUPS has been studied in detail recently by Straub et al. ${ }^{35}$ The method used to obtain an image like Fig. 6 involves selecting an energy window around $E_{F}$. The intensity in this window is represented as a function of parallel momentum $k_{\|}$[function $\left.w\left(k_{\|}\right)\right]$in Fig. 6. Straub et al. ${ }^{35}$ have analyzed how the width of the energetic window (usually limited by the experimental resolution) affects the FS image obtained. A method was proposed to extract from the image the correct $k_{F}$ values,

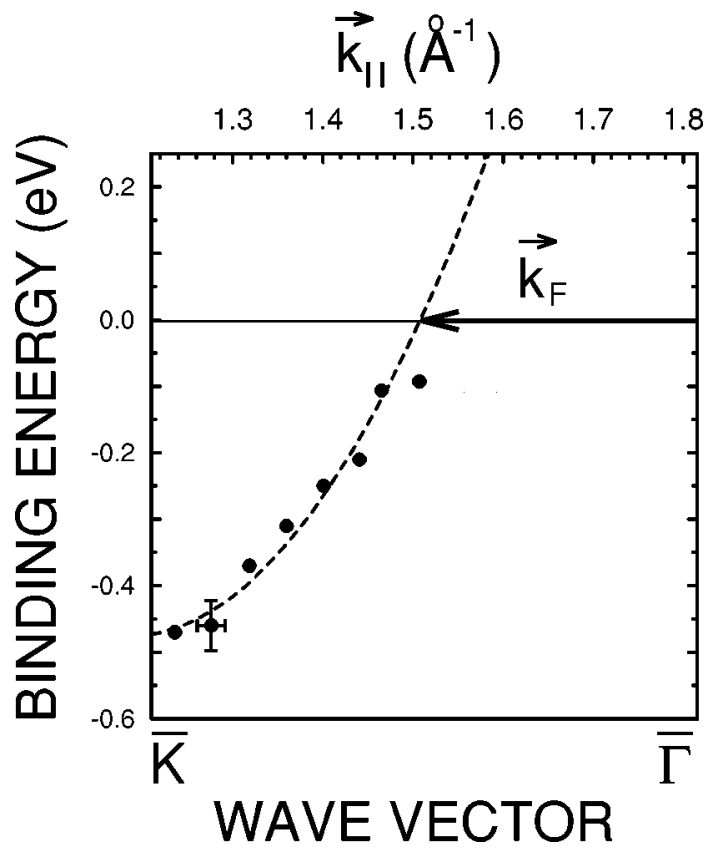

FIG. 5. Dispersion of the surface band vs parallel momentum along $\overline{\Gamma K}$. The value of $k_{F}$ is obtained from the crossing point of the dispersion, fitted to a parabola. From this figure we obtain $k_{F}$ $=1.51 \pm 0.02 \AA^{-1}$.

which can be affected by the experimental resolution. It involves the calculation of the gradient of the FS image, $\left|\nabla_{k_{\|}} w\left(k_{\|}\right)\right|$. This procedure produces two maxima out of each maximum in the original FS image $w\left(k_{\|}\right) \cdot{ }^{35}$ It was found that, out of the two maxima of the gradient $\left|\nabla_{k_{\|}} w\left(k_{\|}\right)\right|$, the one on the unoccupied side of the band crossing $E_{F}$ accurately reflects the value of $k_{F}$. In order to analyze our experimental FS image by this method, we obtained the gradient of the FS image. This is displayed in Fig. 7 (top). The intensity along several $\overline{\Gamma K}$ directions in the first $\left(\bar{\Gamma}_{00} \overline{K_{1}}\right)$ and third $\left(\bar{\Gamma}_{11} \overline{K_{3}}, \bar{\Gamma}_{11} \overline{K_{4}}\right)$ Brillouin zones is shown in the lower panel of Fig. 7. The maxima of the unoccupied side at $\sim 0.3 \AA^{-1}$ correspond to $k_{F}$. The mean value obtained from this figure is $k_{F}=0.30 \pm 0.03 \AA^{-1}$, which coincides with $\bar{M}_{3 \times 3}$ (dashed line). The inner contour in Fig. 7 (top) directly reflects the Fermi line. We note that this kind of analysis (which relates $k_{F}$ to the maximum in the gradient rather than in the direct intensity) would equally apply to the data shown in Fig. 4. If the gradient maxima are used instead of the intensity maxima, also a value of $k_{F}$ $=0.30 \pm 0.03 \AA^{-1}$ is obtained from Fig. 4 .

We conclude that the values of $k_{F}$ obtained from the FS image (intensity plot) are slightly larger than the values needed for a perfect $3 \times 3$ nesting, but the difference is compatible with the experimental accuracy. When the gradient maxima or the surface band dispersion are used, we find a very good agreement with a $3 \times 3$ nesting. Thus, results for the band structure of the RT $\alpha-\sqrt{3}$ phase and the $k_{F}$ value along the $\overline{\Gamma K}$ directions agree well with the calculations shown in Ref. 12. Also, the FS shape found agrees within experimental accuracy with theoretical calculations. ${ }^{12}$ However, the undulated shape of the experimental FS prevents a large nesting along any direction. In particular, the nesting 

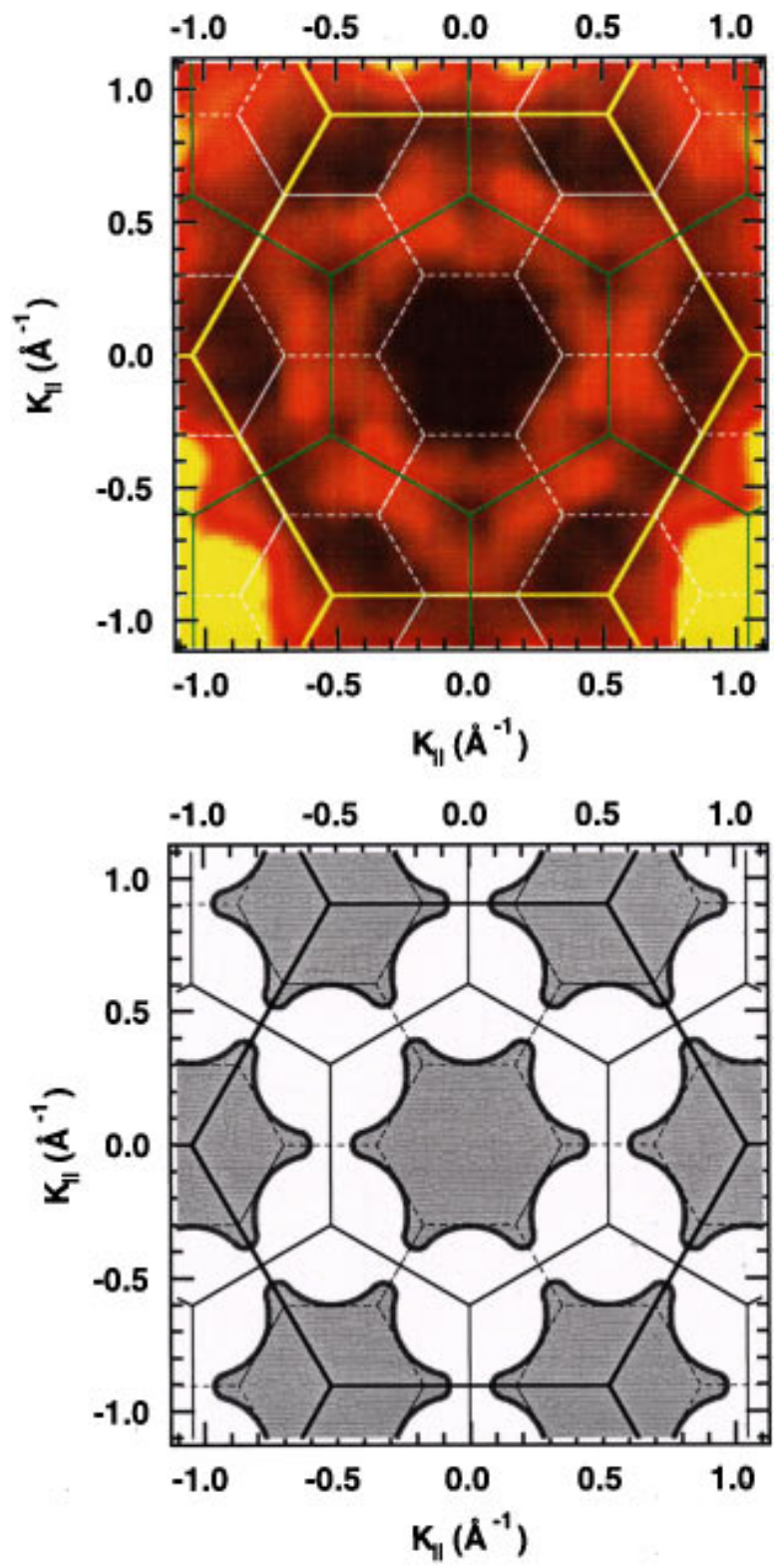

FIG. 6. (Color) Top: Fermi surface of the $\alpha-\sqrt{3}$ phase. The intensity at $E_{F}$ has been converted in a color scale, where lighter color denotes a higher intensity. The Brillouin zones appear in different colors: yellow $(1 \times 1)$, green $(\alpha-\sqrt{3})$, and dashed $(3 \times 3)$. In order to show the whole dynamic range of the image, only the data within the yellow hexagon $(1 \times 1$ Brillouin zone $)$ are scaled. Bottom: schematic representation of the theoretical $\alpha-\sqrt{3}$ Fermi surface (bold contours) in a scale comparable to the top panel. The unshaded area corresponds to occupied states, the only ones observed in the experiment (light areas in top panel). $1 \times 1, \alpha-\sqrt{3}$, and $3 \times 3$ Brillouin zones are in the same scale as top panel.

vectors along the $\overline{\Gamma K}$ directions connect only a reduced percentage of the FS electron density. In short, while $3 \times 3$ vectors join areas of the FS, these areas do not seem to overlap to a large extent, because the undulated shape found reduces the percentage of nested electrons. It might be argued that this percentage could be enough to drive the transition, but this disagrees with the experience accumulated from other CDW materials, where the condition of significant nesting in the FS was always fulfilled. ${ }^{1,4}$ We may recall the case of
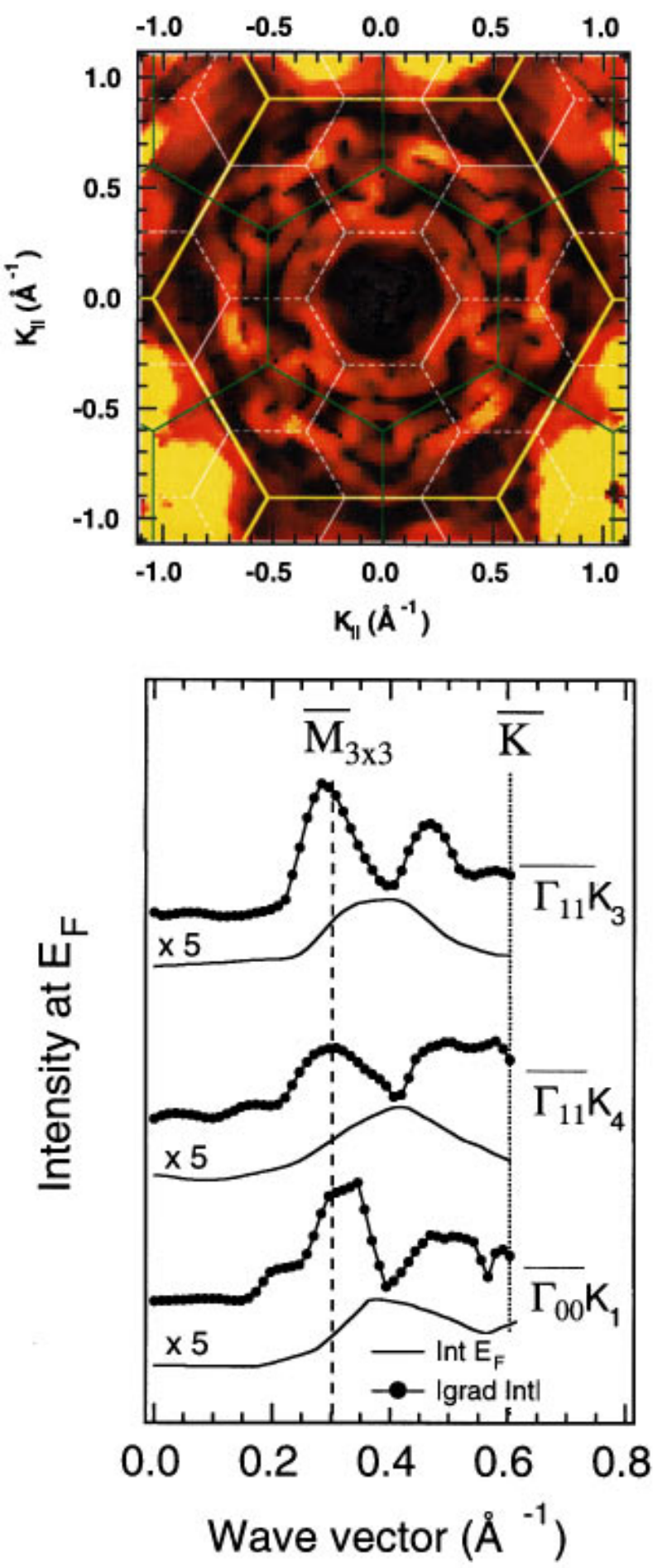

FIG. 7. (Color) Top: gradient of the Fermi surface from Fig. 6. All other details as in Fig. 6. Bottom: intensity vs parallel momentum taken from the top panel along several different $\overline{\Gamma K}$ directions in the first $\left(\bar{\Gamma}_{00} \overline{K_{1}}\right)$ and third Brillouin zones $\left(\bar{\Gamma}_{11} \overline{K_{3}}, \bar{\Gamma}_{11} \overline{K_{4}}\right)$. The maxima at $\sim 0.3 \AA^{-1}$ wave vector reflect the crossing point of a band (Fermi momentum $k_{F}$ ). The intensity along the same direction as measured from Fig. 6 (continuous lines) is shown for comparison.

$\mathrm{NaMo}_{6} \mathrm{O}_{17}$ and $\mathrm{KMo}_{6} \mathrm{O}_{17} \cdot{ }^{39}$ On the other hand, the STM pictures shown in Ref. 12 strongly support the CDW nature of the phase transition. These discrepancies require an investigation of the electronic band structure of the $3 \times 3$ phase, in 


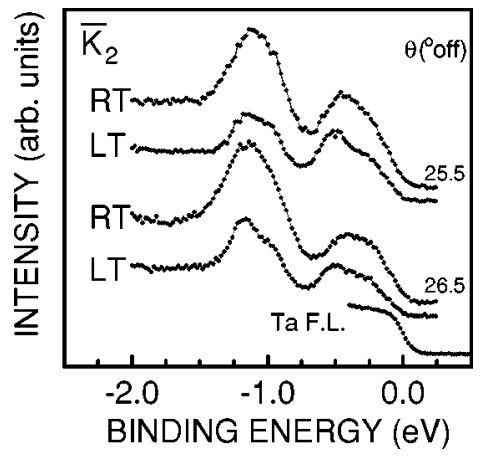

FIG. 8. Detailed view of the band splitting observed around the $\overline{K_{2}}$ point in the $3 \times 3$ phase (spectra at $26.5^{\circ}$ emission angle), and appearance of a new band close to $E_{F}$ (spectra at $25.5^{\circ}$ emission angle). The Fermi level from the Ta shown (bottom spectrum) allows us to estimate the experimental resolution.

order to test the LDA predictions ${ }^{12}$ and the nature of the bands at LT.

\section{IV. $3 \times 3$ PHASE: RESULTS AND DISCUSSION}

As the temperature decreases the LEED pattern reverts to $3 \times 3$. The formation of a different reconstruction with a larger surface unit cell produces a different surface electronic band structure. The simplest approach to obtain the new band structure is to fold the bands over the $3 \times 3$ unit cell, as shown in Ref. 12. Along the $\overline{\Gamma K}$ direction from Fig. 1, $\bar{\Gamma}$ and $\bar{K}$ points become $\bar{\Gamma}_{3 \times 3}$ points, while the $\bar{M}$ points and the points halfway between the $\bar{\Gamma}$ and $\bar{K}$ points become $\bar{M}_{3 \times 3}$ points (see also upper and lower axis of Fig. 3). Concerning $S_{2}$, folding effects should produce an additional surface band at $\bar{\Gamma}_{11}$, which becomes equivalent to $\overline{K_{2}}$. This band is not observed in the experiment, probably because of the smaller bulk band gap at this reciprocal space point. Also $S_{1}$, when folded over the $3 \times 3$ cell, should produce an additional band at $\bar{\Gamma}_{11}$. This is indeed observed as a faint feature at $\sim 0.9 \mathrm{eV}$ BE close to $\bar{\Gamma}_{11}$, but only for the upper branch of the $S_{1}$ band. Thus, note that the peak at $1.2 \mathrm{eV} \mathrm{BE}$ in $\bar{\Gamma}_{11}$ is not affected by the phase transition. Since the symmetry of both the upper and lower branches of $S_{1}$ at the $\bar{M}_{1}$ points at RT is not necessarily the same, the bulk bands may mix more efficiently with one of them. An additional interesting feature of $S_{1}$ is the following: the two $S_{1}$ branches are degenerate at $\mathrm{RT}$ in the $\bar{K}_{1}$ and $\bar{K}_{2}$ points (Fig. 3 , top). The degeneracy is lifted at LT, when a band gap opens in these two points (compare upper and lower panels of Fig. 3). The splitting at $\bar{K}_{2}$ is shown in Fig. 8, spectra at $26.5^{\circ}$ emission angle.

Additional important effects are observed at LT. A new dispersing surface band (with minima at $\bar{\Gamma}_{3 \times 3}$ points) is detected at LT in the vicinity of $E_{F}$ (compare upper and lower panels of Fig. 3). The new band approximately reproduces the dispersion of $S_{2}$, but is shifted to lower BE by $\sim 0.2 \mathrm{eV}$ at the $\bar{\Gamma}_{3 \times 3}$ points. It exhibits an obvious $3 \times 3$ periodicity, and it is observed in three different $\bar{\Gamma}_{3 \times 3}$ points along the

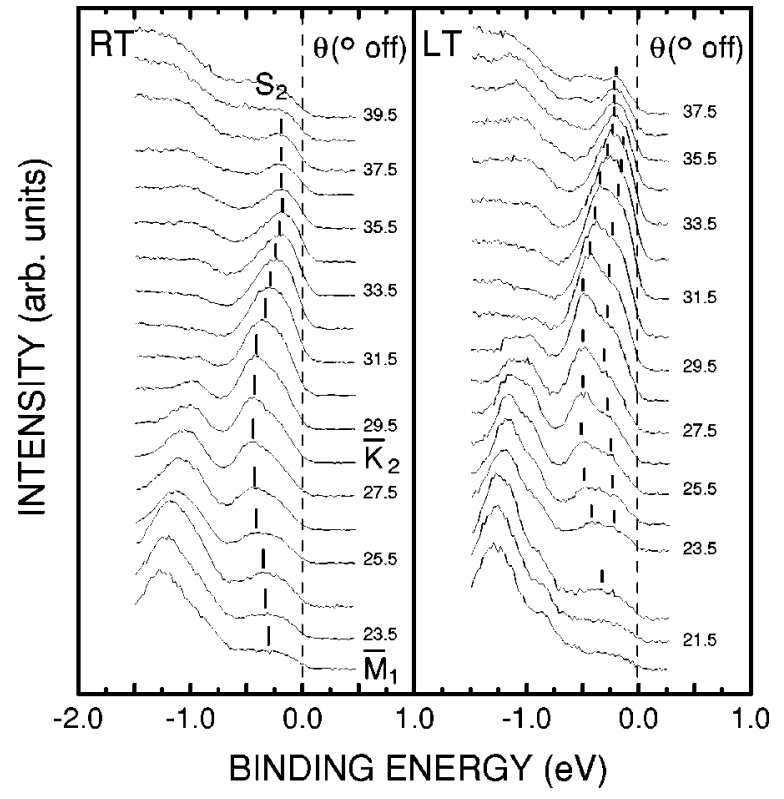

FIG. 9. Photoemission spectra as a function of emission angle along the $\overline{\Gamma K}$ direction ( $h \nu=32 \mathrm{eV})$ at RT (left) and LT (right). The data correspond to the angular range around $\bar{K}_{2}$. Note the splitting of $S_{2}$ at LT.

$\overline{\Gamma K}$ direction (see Fig. 3, bottom). The appearance of the new band is also shown in Fig. 8, spectra at $25.5^{\circ}$ emission angle, which correspond to $\bar{K}_{2}$ for $\sim 0.2 \mathrm{eV} \mathrm{BE}$. The spectra corresponding to this angular range around $\bar{K}_{2}$ are reproduced in more detail ( $1^{\circ}$ steps) in Fig. 9, where the band splitting can be monitored over a wider range around $\bar{K}_{2}$.

The band found is not predicted in density-functional calculations, ${ }^{12}$ which, on the other hand, reproduce quite well the electronic band structure of the $\alpha-\sqrt{3}$ phase, as shown before. One possible explanation for this new surface band found could be a partial occupation of an (initially) unoccupied band. The first empty band ${ }^{12}$ lies indeed very close to $E_{F}$ both at the $\bar{\Gamma}_{3 \times 3}$ and $\bar{M}_{3 \times 3}$ points. However, the dispersion observed in the experiment does not agree with the dispersion expected for the first empty band, and the new surface band found is not seen around $\bar{M}_{1}$, as expected for the surface empty band. ${ }^{12}$ A second possible explanation is that the two quasidegenerate bands predicted along the $\overline{\Gamma M}$ directions ${ }^{12}$ present a much higher splitting than predicted, or that they are split due to other kinds of effects. In any case, the conclusion is that existing LDA calculations for the 3 $\times 3$ phase do not explain the bands found at LT. Taking into account the difficulty of LDA to account for electronelectron interactions (exchange-correlation energy), this result could be an indication of the important role played by these effects in the explanation of the electronic properties found at LT. Alternatively, an incorrect structure would have a drastic effect on the calculated band structure, and could also be behind the disagreement found. Thus, a final answer to the origin of the splitting can be found only when the atomic structure of the LT phase is known. ${ }^{23}$ However, the data exhibit features that deserve some comments. We note that RT spectra from Fig. 8 show rather broad peaks. Both 


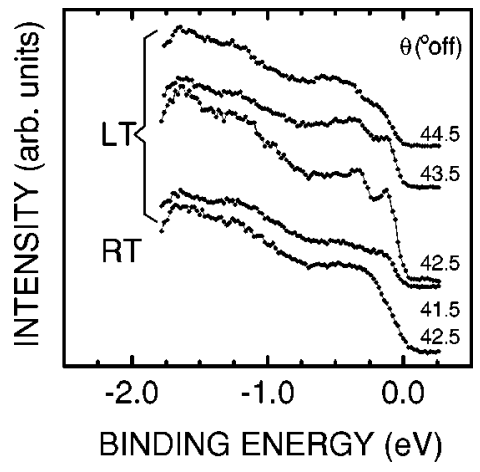

FIG. 10. Valence-band spectra taken at RT and LT around the $\bar{\Gamma}_{11}$ point, corresponding to the $\alpha-\sqrt{3}$ and the $3 \times 3$ phases, respectively. A narrow, intense peak appears close to the Fermi level as the $3 \times 3$ phase is stabilized.

the band found in the vicinity of $E_{F}$ and the splitting of $S_{1}$ at the $\bar{K}$ points are detected as shoulders in the LT peaks. This is not an effect of the experimental resolution (see also below, and the Fermi level from Ta shown in Fig. 8, bottom). It rather reflects an intrinsic width of the peaks. If we accept that the phase transition involves some kind of temperaturedriven buckling of the $\mathrm{Pb}$ atoms to form a $3 \times 3$ structure, the temperature-dependent splitting could be interpreted in the way proposed in Ref. 40 for the valence band and Ref. 41 for the core levels of similar $\sqrt{3}$ structures, i.e., as a consequence of the existence of two kinds of $\mathrm{Pb}$ atoms, whose vibrational movement is stabilized at LT. The broader structure found at RT would reflect the envelope between the different contributions coming from the vibrational movement of $\mathrm{Pb}$ atoms, which would be frozen at LT. This possible explanation cannot be proved on the basis of the electronic structure data alone, and detailed information on the atomic structure of the $3 \times 3$ phase is required.

The results shown demonstrate that there is a significant effect of the phase transition on the electronic band structure. These results can also shed light on the role of electronelectron interactions to stabilize the LT phase. In fact, correlation effects have been invoked to explain the electronenergy-loss spectroscopy results ${ }^{12}$ at LT, which show the opening of a band gap. ${ }^{12,21}$ We will address this topic elsewhere. ${ }^{23}$ Both surface states found exhibit bandwidths of $\sim 0.4 \mathrm{eV}$. This means a significant degree of delocalization for these electrons. On the other hand, the spectra shown in Fig. 8 (top) leave little doubt about the importance of changes in the electronic structure that take place at the same time as the phase transition. As the temperature decreases, the spectral density in the region closer to $E_{F}$ rearranges itself to produce a narrow peak at $0.1 \mathrm{eV} \mathrm{BE}$ (see Fig. 10). This effect is observed with high intensity in the vicinity of the $\bar{\Gamma}_{11}$ point (spectrum at $42.5^{\circ}$ emission angle in Fig. 10 ). The width of the peak, much smaller than any other feature observed at this interface (compare for instance with Fig. 8, bottom), suggests that it is related to localized electrons in a state where fluctuations are considerably reduced, probably because of the wave-function symmetry at $\bar{\Gamma}_{11}$. This peak occurs exclusively in the $3 \times 3$ phase, and is observed in a fully reversible way when the phase transition is crossed upon decreasing the temperature. The simplest explanation is that the charge transfer giving rise to the new periodicity found at LT is related with a partial electron localization. Delocalized electrons in the $\alpha-\sqrt{3}$ phase become partly localized at LT in a state where exchange-correlation effects are enhanced. In this case the narrow peak at $\bar{\Gamma}_{11}$ could reflect the formation of a lower Hubbard band.

\section{CONCLUSIONS}

We conclude that the $\alpha-\sqrt{3}$ reconstruction presents a band structure with dispersing surface peaks, in agreement with LDA theoretical predictions. The phase transition to the $3 \times 3$ phase takes place concomitantly with changes in the electronic band structure. Band folding effects are also observed at the precise regions of the reciprocal space that correspond to the $3 \times 3$ periodicity. The observation of a band splitting at LT giving rise to dispersing bands centered at the $\bar{\Gamma}_{3 \times 3}$ points indicates that electronic states involved in the transition are delocalized. A possible structural origin for the splitting is proposed. Finally, the appearance of a different surface band with a $3 \times 3$ periodicity is of relevance in order to understand the role of Fermi-surface nesting in the transition. While nesting and CDW stabilization has been invoked as the mechanisms behind the transition, in view of STM results, ${ }^{12}$ the experimental Fermi surface found exhibits only limited nested areas.

\section{ACKNOWLEDGMENTS}

We thank E. Tosatti, G. LeLay, and F. Flores for discussions, J. Osterwalder and his group for their assistance and support in the construction of the manipulator, and R. Stumpf for communicating to us unpublished results. This work was financed by DGICYT (Spain) under Grant Nos. PB-94-1527 and PB-94-0022-C02-01. The access of A.M. and E.G.M. to LURE, Centre Universitaire Paris-Sud, was supported through the Large Scale Facilities program of the European Union. A.M. thanks Eusko Jaurlaritza for financial support.
${ }^{1}$ For a recent review see G. Grüner, Density Waves in Solids (Addison-Wesley, Reading, MA, 1994).

${ }^{2}$ E. Tosatti in Electronic Surface and Interface States in Metallic Systems, edited by E. Bertel and M. Donath (World Scientific, Singapore, 1995), and references therein.
${ }^{3}$ R. E. Peierls, Quantum Theory of Solids (Oxford University Press, New York, 1955).

${ }^{4}$ E. Tosatti, Festkoerperprobleme XV, 113 (1975).

${ }^{5}$ S. D. Kevan, J. Electron Spectrosc. Relat. Phenom. 75, 175 (1995) and references therein. 
${ }^{6}$ E. Tosatti, Solid State Commun. 25, 637 (1978).

${ }^{7}$ M. K. Debe and D. A. King, J. Phys. C 10, L303 (1977).

${ }^{8}$ T. E. Felter, R. A. Barker, and P. J. Estrup, Phys. Rev. Lett. 38, 1138 (1977).

${ }^{9}$ X. W. Wang and W. Weber, Phys. Rev. Lett. 58, 1452 (1987).

${ }^{10}$ K. E. Smith, G. S. Elliott, and S. D. Kevan, Phys. Rev. B 42, 5385 (1990).

${ }^{11}$ F. M. Hoffmann, B. N. J. Persson, W. Walter, D. A. King, C. J. Hirschnagl, and G. P. Williams, Phys. Rev. Lett. 72, 1256 (1994).

${ }^{12}$ J. Carpinelli, H. Weitering, E. W. Plummer, and R. Stumpf, Nature (London) 381, 398 (1996).

${ }^{13}$ T. Ichikawa, Solid State Commun. 46, 827 (1983).

${ }^{14}$ R. Feidenhans'1, J. S. Pedersen, M. Nielsen, F. Grey, and R. L. Johnson, Surf. Sci. 178, 927 (1986).

${ }^{15}$ L. Seehofer, D. Daboue, G. Falkenberg, and R. L. Johnson, Surf. Sci. 307-309, 698 (1994).

${ }^{16}$ L. Seehofer, D. Daboul, G. Falkenberg, and R. L. Johnson, Surf. Sci. 314, L879 (1994).

${ }^{17}$ A second $\sqrt{3} \times \sqrt{3} R 30^{\circ}$ reconstruction $\left(\beta-\sqrt{3} \times \sqrt{3} R 30^{\circ}\right)$ phase exists at higher coverage. We restrict ourselves in this paper to $\Theta_{\mathrm{Pb}}=\frac{1}{3}$ ML, i.e., the $\alpha$ phase.

${ }^{18}$ P. Aebi, J. Osterwalder, P. Schwaller, L. Schlapbach, M. Shimoda, T. Mochiku, and K. Kadowaki, Phys. Rev. Lett. 72, 2757 (1994).

${ }^{19}$ J. Avila, C. Casado, M. C. Asensio, J. L. Perez, M. C. Muñoz, and F. Soria, J. Vac. Sci. Technol. A 13, 1501 (1995).

${ }^{20}$ N. L. Saini, J. Avila, A. Bianconi, A. Lanzara, M. C. Asensio, S. Tajima, G. D. Gu, and N. Kashizuka, Phys. Rev. Lett. 79, 3467 (1997).

${ }^{21}$ A. Goldoni, C. Cepek, and S. Modesti, Phys. Rev. B 55, 4109 (1997).

${ }^{22}$ K. Würde, P. Krüger, A. Mazur, and J. Pollmann, Surf. Rev. Lett. (to be published).

${ }^{23}$ A. Mascaraque, J. Avila, M. C. Asensio, and E. G. Michel (unpublished).

${ }^{24}$ J. A. Carlisle, T. Miller, and T. C. Chiang, Phys. Rev. B 47, 3790 (1993).
${ }^{25}$ G. LeLay, V. Yu. Aristov, L. Seehofer, T. Buslaps, R. L. Johnson, M. Gothelid, M. Hammar, U. O. Karlsson, S. A. Flodström, R. Feidenhans'l, M. Nielsen, E. Findeisen, and R. I. G. Uhrberg, Surf. Sci. 307-309, 280 (1994).

${ }^{26}$ D. R. Heslinga, H. H. Weitering, D. P. van der Werf, T. M. Klapwijk, and T. Nibma, Phys. Rev. Lett. 64, 1589 (1990).

${ }^{27}$ G. LeLay, K. Hricovini, and J. Bonnet, Appl. Surf. Sci. 41/42, 25 (1989).

${ }^{28}$ J. A. Carlisle, T. Miller, and T. C. Chiang, Phys. Rev. B 47, 10 342 (1993).

${ }^{29}$ L. Seehofer, G. Falkenberg, and R. L. Johnson, Surf. Sci. 290, 15 (1993).

${ }^{30}$ C. J. Karlsson, E. Landemark, Y. C. Chao, and R. I. G. Uhrberg, Phys. Rev. B 45, 6321 (1992).

${ }^{31}$ A. Mascaraque, J. Avila, M. C. Asensio, and E. G. Michel, Surf. Sci. (to be published).

${ }^{32}$ J. E. Northrup, Phys. Rev. Lett. 53, 683 (1984).

${ }^{33}$ B. P. Tonner, H. Li, M. J. Robrecht, M. Onellion, and J. L. Erskine, Phys. Rev. B 36, 989 (1987).

${ }^{34}$ Data obtained from $\bar{K}_{2} \bar{\Gamma}_{11} \bar{K}_{3}$, where the intensity of $S_{2}$ is highest.

${ }^{35}$ Th. Straub, R. Claessen, P. Steiner, S. Hüfner, V. Eyert, K. Friemelt, and E. Bucher, Phys. Rev. B 55, 13473 (1997).

${ }^{36} k_{\|}$is conserved in the photoemission process. See F. J. Himpel, Adv. Phys. 32, 1 (1983) for details.

${ }^{37}$ The origin of this feature lies in the enhanced intensity detected at $\sim 0.2 \mathrm{eV} \mathrm{BE}$ at $\bar{\Gamma}_{11}$, which is probably related with the other phenomena found in this area at LT (see Sec. IV).

${ }^{38}$ The intensity enhancement in the two bottom corners of Fig. 6 (top) comes from a larger bulk band-gap projection and matrix element effects in the second Brillouin zone (Ref. 23).

${ }^{39}$ G. H. Gweon, J. W. Allen, J. A. Clack, Y. X. Zhang, D. M. Poirier, P. J. Benning, C. G. Olson, J. Marcus, and C. Schlenker, Phys. Rev. B 55, R13 353 (1997).

${ }^{40}$ H. H. Weitering, X. Shi, P. D. Johnson, J. Chen, N. J. DiNardo, and K. Kempa, Phys. Rev. Lett. 78, 1331 (1997).

${ }^{41}$ M. Göthelid, M. Björkqvist, T. M. Grehk, G. Le Lay, and U. O. Karlsson, Phys. Rev. B 52, R14 352 (1995). 